\title{
Targeting RAD50 increases sensitivity to radiotherapy in colorectal cancer cells
}

\author{
C. CHEN ${ }^{1, *}$, Y. WANG ${ }^{2, *}$, J.F. MEI ${ }^{3}$, S.S. $\mathrm{LI}^{2}$, H.X. XU' ${ }^{3}$, H.P. XIONG ${ }^{2}$, X.H. WANG ${ }^{3, *}$, X. HE ${ }^{1, *}$
}

${ }^{1}$ Department of Radiotherapy, Nanjing Medical University Affiliated Cancer Hospital, Cancer Institute of Jiangsu Province, Jiangsu Cancer Hospital, Baiziting 42, Nanjing 210009, China; ${ }^{2}$ Department of Oncology, Traditional Chinese Medical Hospital of Siyang County, 15 Jiefangbei Road, Siyang County, Jiangsu, 223700, China; ${ }^{3}$ Department of chemotherapy, Nanjing Medical University Affiliated Cancer Hospital, Cancer Institute of Jiangsu Province, Jiangsu Cancer Hospital, Baiziting 42, Nanjing 210009, China

*Correspondence: hexia1505@163.com,wangxiaohua8110@sina.com

"Contributed equally to this work.

Received February 19, 2017/ Accepted May 29, 2017

\begin{abstract}
Radiotherapy resistance remains the major factor limiting the radiotherapy efficacy in colorectal cancer. The Mre11RAD50-Nbs1 (MRN) complex is known to play a critical role in the DNA double strand breaks (DSBs) repair pathways and thus facilitates radioresistance. Targeting MRN function can sensitize cancer cells to irradiation in some malignancies. In this study, we stably knocked down RAD50 protein in colorectal cancer (CRC) cell lines, HCT116 and DLD1, and evaluated their response to irradiation as well as the DSB repair dynamics. We observed that downregulation of RAD50 sensitized CRC cells to irradiation with reduction in DSB repair efficiency after exposure to irradiation. In addition, RAD50 was found to be upregulated in CRC cancerous tissue samples compared to non-cancerous adjacent tissues (NATs) and in patients who were resistant to RT. Elevated RAD50 expression was associated with poor patient survival in CRC. In conclusion, targeting RAD50 can serve as an efficient strategy to sensitize CRC cells to irradiation. RAD50 protein may be used as a biomarker for patient survival in CRC.
\end{abstract}

Key words: RAD50, colorectal cancer, DSB repair, radiosensitivity

Radiotherapy (RT) is one of the major approaches to treat multiple cancer types, including lung, colorectal, breast and prostate cancer [1]. Radiation, often combined with chemotherapy, may be used before or after surgical resection of tumors to decrease the risk of recurrence. Radiation exerts cytotoxicity through induction of sustained DNA double-strand breaks (DSB) [2]. However, enhanced DNA repair capacity in tumor cells has been considered as the major cause of radioresistance and thus unsuccessful treatment. Inhibition of DSB repair through targeting repair proteins can sensitize tumor cells to irradiation (IR) and increase radiotherapy efficacy. Therefore, identification of novel targets as RT sensitizers is imperative $[3,4]$.

In response to DSBs, cells activate the so-called DNA damage response (DDR) that activates the DNA damage checkpoint, blocks the cell cycle, and mediates DNA repair in a highly coordinated manner. In case DSBs are left unrepaired, cells undergo apoptosis to prevent the transmission of severe DNA damage to offspring. The MRN complex facilitates the two DSBs repair pathways- homologous recombination (HR) repair and non-homologous end joining (NHEJ) in humans. This complex has critical functions in several aspects of DDR, including DSBs detection, subsequent signaling, cell cycle regulation and telomere maintenance $[5,6]$. Due to its crucial function in DDR, targeting the protein components of this complex represents an effective strategy to sensitize tumor cells to RT.

The human RAD50 gene is located on chromosome $5 \mathrm{q} 31$ and encodes a $153 \mathrm{kDa}$ protein. During DSBs repair, Mre11 and RAD50 form functional Mre $11_{2} / \mathrm{RAD}_{20}$ heterotetramers that bind to either DNA ends or sister chromatids and are associated with other heterotetramers to initiate DSBs repair [7]. It is essential for all the aspects of MRN complex function. Targeting RAD50 has been reported to sensitize tumor cells to RT in nasopharyngeal carcinoma and squamous cell carcinoma $[8,9]$.

In this study, we investigated the role of RAD50 in mediating radiosensitivity in CRC and its potential clinical significance. 


\section{Materials and methods}

Cell culture. Human colorectal cancer cells HT29 and HCT116 (ATCC) were cultured in basal medium supplemented with $10 \%$ serum at $37^{\circ} \mathrm{C}$ and $5 \% \mathrm{CO}_{2}$. These cells were tested mycoplasma free.

Viral transductions and stable selections. For lentivirus production, $1 \mu \mathrm{g}$ of shRNA (Santa Cruz Biotechnology, sc-37397-SH), together with $0.4 \mu \mathrm{g}$ pMD2G and $0.6 \mu \mathrm{g}$ psPAX2 were transfected into 293FT cells using Effectene reagent (Qiagen, Valencia, CA, USA). Viral supernatants were collected 48 hours afterwards, cleared through a $0.45 \mu \mathrm{m}$ filter and added onto cells containing $4 \mu \mathrm{g} / \mathrm{mL}$ polybrene (Sigma, St. Louis, MO, USA) and selected under puromycin for 7 days.

Colony formation assay. After exposure to $\gamma$-radiation, cells were trypsinized and seeded in 6-well plates at a density of 1,000 cells/well in triplicates and incubated for 1 week. Cells were washed with PBS three times, fixed with $4 \%$ paraformaldehyde for $15 \mathrm{~min}$, and stained with $0.1 \%$ crystal violet (Sigma-Aldrich, USA) solution for $30 \mathrm{~min}$. Then the colonies were washed with PBS three times. Colony formation efficiency was calculated as colony number divided by 1,000 and normalized to control cells.

Immunoblot analysis. Total cell lysates were harvested from cells in Laemmli S.D.S reducing buffer $(50 \mathrm{mM}$ Tris- $\mathrm{HCl}$ (pH 6.8), 2\% S.D.S, and 10\% glycerol), boiled and resolved on a gradient polyacrylamide gel, and then transferred onto polyvinylidinefluoride membrane. After incubation with antibodies against RAD50 (Abcam, Cambridge, MA, USA), and $\beta$-actin (Sigma-Aldrich, USA) for 1 hour, the blots were incubated with anti-rabbit or anti-mouse IgG secondary antibody (1:5000, Santa Cruz Biotechnology, Dallas, USA) and developed with Femto Chemiluminescent Substrate Kit (Thermo Scientific, Grand Island, USA).

Caspase 3/7 activity. Control or RAD50 knockdown cells were exposed to 4 Gy of $\gamma$-radiation and followed by CaspaseGlo 3/7 assay according to the manufacturer's instructions (Promega, Madison, WI, USA).

$\boldsymbol{\gamma}$-H2AX assay. After exposure to $2 \mathrm{~Gy}$ of $\boldsymbol{\gamma}$-radiation, cells were fixed using $4 \%$ paraformaldehyde for $10 \mathrm{~min}$, permeabilized in PBS with $0.5 \%$ TritonX-100 for $5 \mathrm{~min}$ and incubated with blocking solution for $60 \mathrm{~min}$, followed by $\gamma$-H2AX antibody (1:100, Abcam, ab18311) incubation for an hour. After incubation with AlexaFluor-568 labeled secondary antibodies (1:400, Invitrogen, Carlsbad, CA, USA), slides were mounted and cells were analyzed under a fluorescence microscope (Axiovert 200M, CarlZeiss, Gottingen, Germany).

Patients and treatments. The study was approved by the Ethical Committee of Nanjing Medical University Affiliated Cancer Hospital, and informed consent was obtained from all patients. Resection specimens and matched non-cancerous adjacent tissues (NATs) were collected from CRC patients with primary cancer who were subjected to surgery only between January 2013 and July 2015 at Nanjing Medical University Affiliated Cancer Hospital. All excised tissues were immedi- ately snap-frozen in liquid nitrogen and then were stored at $-80^{\circ} \mathrm{C}$. A total of 36 pairs of CRC tissues were examined in the current study. In addition, 44 patients diagnosed with locally advanced CRC were recruited between March 2013 and December 2015. Biopsy samples were obtained before radiotherapy was delivered to these patients involving a total dose of 45.0-50.4 Gy of IR over five weeks. Radical surgery was performed 2-3 weeks after completing radiotherapy at Nanjing Medical University Affiliated Cancer Hospital. The prognosis was evaluated for all of the patients in October 2015. Overall survival was defined as the time from onset of cancer till death or till censoring on the last follow-up date.

Tumor regression grading (TRG). Pathological analysis of radiotherapy response was evaluated according to $\mathrm{H}$ and $\mathrm{E}$ staining using the TRG system according to Dworak [10] as follows: TRG 0, no regression; TRG 1, dominant tumor mass with obvious fibrosis in $\leq 25 \%$ of the tumor mass; TRG 2 , dominant tumor mass with obvious fibrosis in $26-50 \%$ of the tumor mass; TRG 3, dominant fibrosis outgrowing the tumor mass; and TRG 4, no viable tumor cells (only a fibrotic mass). Histological and TRG evaluations were performed by two pathologists blinded to the clinical and pathological features associated with the specimens.

Isolation of total RNA and quantitative RT-PCR. Total RNAs were extracted with Qiagen RNeasy Mini kit (Qiagen, Hilden, Germany) according to the manufacturer's protocol, and then mRNAs was reverse transcribed to cDNA using PrimeScript $^{\text {TM }}$ RT Master Mix Perfect Real Time (TaKaRa, Dalian, China). qPCR reaction was performed in triplicates on the Bio-Rad Fluorescence Thermal Cycler (Bio-Rad Laboratories, CA, USA) as follows: $95^{\circ} \mathrm{C}$ for $10 \mathrm{~min}$; amplification for 36 cycles, with denaturation at $95^{\circ} \mathrm{C}$ for $30 \mathrm{sec}$; annealing at $56^{\circ} \mathrm{C}$ for $30 \mathrm{sec}$; and elongation at $72^{\circ} \mathrm{C}$ for $30 \mathrm{sec}$, followed by a final extension at $72^{\circ} \mathrm{C}$ for $10 \mathrm{~min}$. GAPDH mRNA level was used for normalization.

Statistical analysis. Quantitative data represent mean \pm SD. Statistical significance was evaluated by the Student t test. For patient sample data, statistical analysis was performed using the SPSS statistical package, version 16.0 (SPSS Inc., Chicago, IL, USA). The Mann-Whitney U or Wilcoxon test was used to compare RAD50 mRNA expression level in unpaired or paired tumor tissue samples versus NATs, respectively. The Pearson's chi-squared test was used to assess the association between RAD50 mRNA level and response to radiotherapy. Kaplan-Meier curves were compared using log-rank test. The p-value of two-sided less than 0.05 was considered statistically significant.

\section{Results}

RAD50 modulates radiosensitivity in CRC cells. To investigate whether RAD50 plays a role in regulating radiosensitivity in CRC, we stably knocked down RAD50 in CRC cell lines, HCT116 and HT29. Each of the two shRNAs 
efficiently reduced RAD50 protein level in both cell lines (Figure 1A and B). Downregulation of RAD50 led to a significant sensitization to radiation in both HCT116 and HT29 cells in colony formation assay (Figure 1C and D), suggesting the direct involvement of RAD50 in regulating radiosensitivity. Consistently, CTG assay confirmed that knockdown of RAD50 further reduced number of surviving cells after exposure to IR compared to Nsi cells, decreasing surviving fraction from $\sim 58.7 \%$ to $\sim 35 \%$ in HCT116 cells and $\sim 62 \%$ to $\sim 32 \%$ in HT29 cells, respectively (Figure $2 \mathrm{~A}$ ). To assess whether there was an increased induction of apoptosis, we measured the caspase 3/7 activity in control and RAD50 knockdown HCT116 and HT29 cells after exposure to 4 Gy of irradiation. We observed that downregulation of RAD50 resulted in $\sim 3$-fold increase in caspase $3 / 7$ activity, indicating enhanced induction of apoptosis (Figure 2B).
A

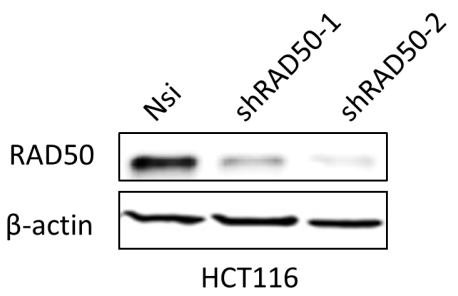

C

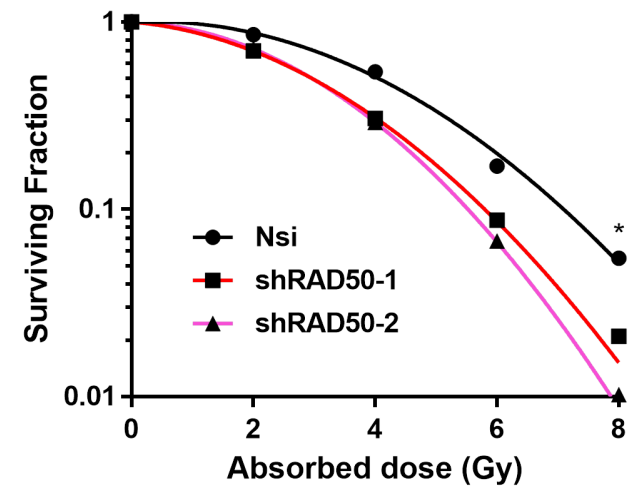

B
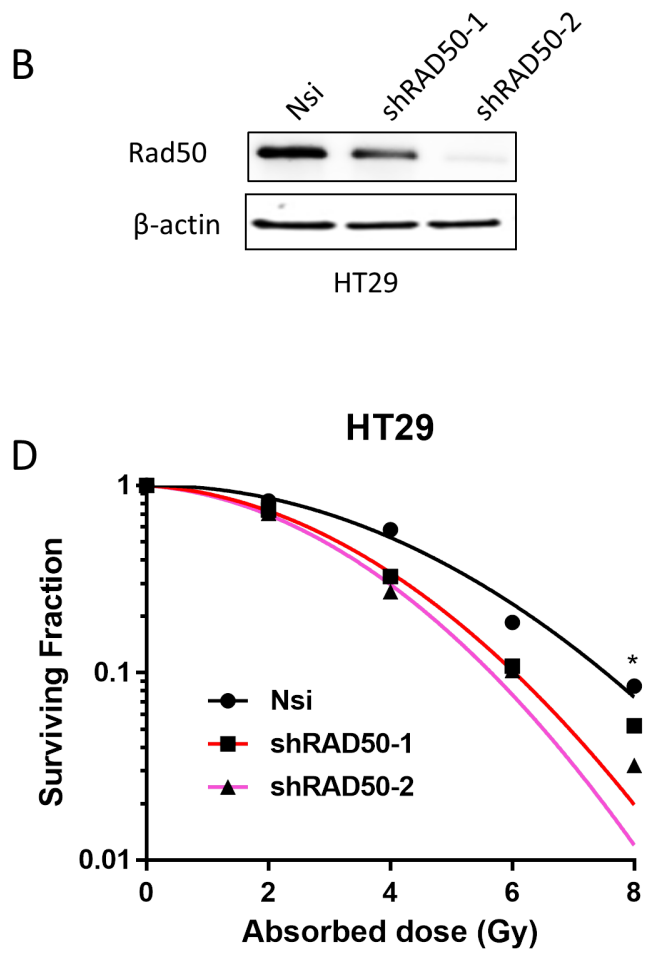

Figure 1. RAD50 modulates radiosensitivity in CRC cells. A and B. Western blot analysis of RAD50 protein level in HCT116 (A) and HT29 (B) cells expressing non-silencing (Nsi) shRNA or RAD50 shRNA. C and D. Clonogenic survival in HCT116 (C) and HT29 (D) cells expressing non-silencing (Nsi) shRNA or RAD50 shRNA after exposure to $2,4,6$ or 8 Gy of $\gamma$-radiation. Survival was normalized to unirradiated control cells. Data represent mean \pm SD. ${ }^{*}, \mathrm{p}<0.05$.

A

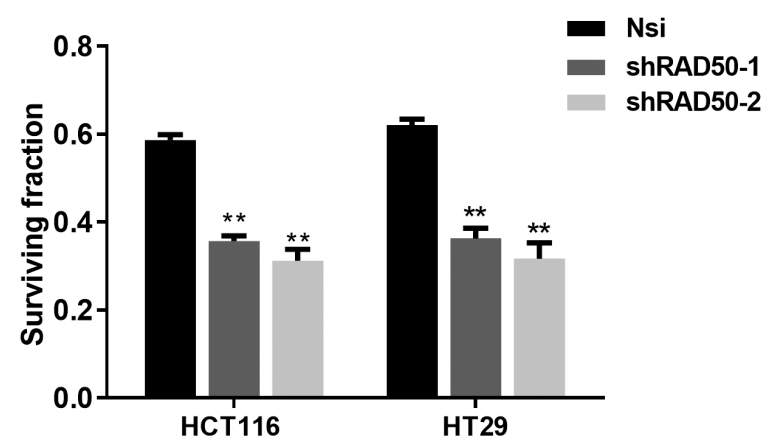

B

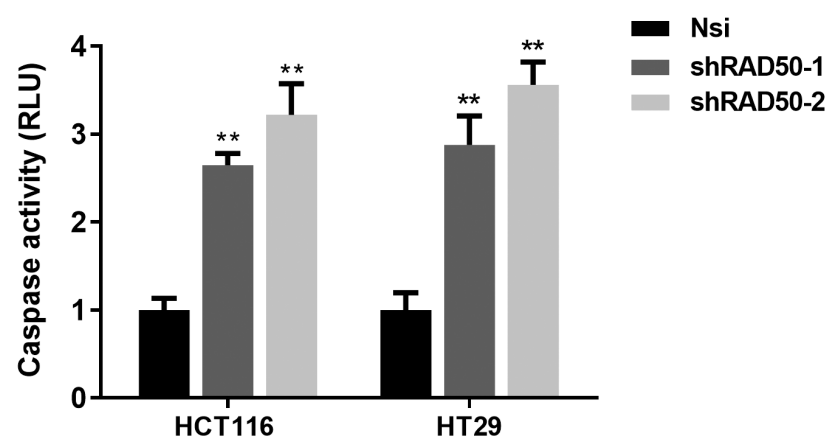

Figure 2. RAD50 affects cell survival and apoptosis after IR exposure. A. Surviving fraction of HCT116 and HT29 cells expressing non-silencing (Nsi) shRNA or RAD50 shRNA after exposure to 4 Gy of $\gamma$-radiation. Student's t-test, ${ }^{* *}, p<0.01$. B. Caspase $3 / 7$ activity in cells as described in A. Data represent mean $\pm \mathrm{SD}$. Student's t-test, ${ }^{* *}, \mathrm{p}<0.01$. 
Effects of RAD50 knockdown on $\gamma$-H2AX foci formation after IR exposure. It is known that $\mathrm{H} 2 \mathrm{AX}$ is phosphorylated rapidly after formation of DSB and aggregates at the site of DSB, serving as an important method to detect DSB repair efficiency. To further evaluate the role of RAD50 in radioresistance, DSBs were assessed and quantified by $\gamma$-H2AX foci staining 0.5, 1, 3 and 6 hours post IR. Compared with Nsi cells, significantly more $\gamma$-H2AX foci were present 6 hours after irradiation in RAD50 knockdown cells in both HCT116 and HT29 cell lines (Figure 3). These results suggested that reduced expression of RAD50 compromised DDR efficiency, thus conferring sensitivity to IR.

RAD50 expression level is positively associated with radioresistance in $\mathrm{CRC}$ patients. To evaluate clinical significance of RAD50 in CRC patients, mRNA levels of RAD50 were measured in $36 \mathrm{CRC}$ tissue samples and matched NAT samples using qPCR. As shown in Figure 4A, expression levels of RAD50 were significantly increased in cancerous tissue $(\mathrm{p}<0.0001)$. In addition, RAD50 mRNA levels were found to be significantly higher in tumors from 23 CRC patients who had poor response to radiotherapy compared to 21 patients who showed poor response $(\mathrm{p}<0.0001)$ (Figure 4B). Moreover, we observed a significant association between mRNA level of RAD50 and radiotherapy response $(\mathrm{p}=0.001 ;$ Table 1$)$.

\section{Discussion}

Cancer cells activate a number of signaling pathways to enhance DNA-repair capacity and promote cell survival which leads to radioresistance and limited efficacy of RT [11].
A

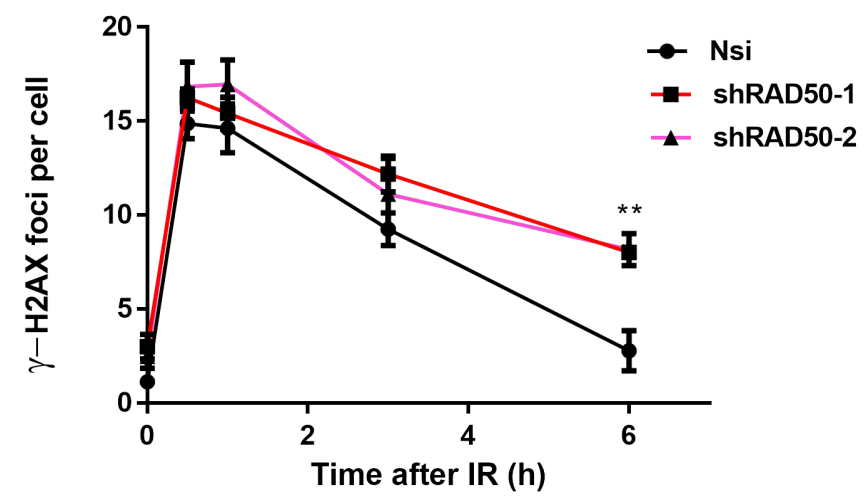

B

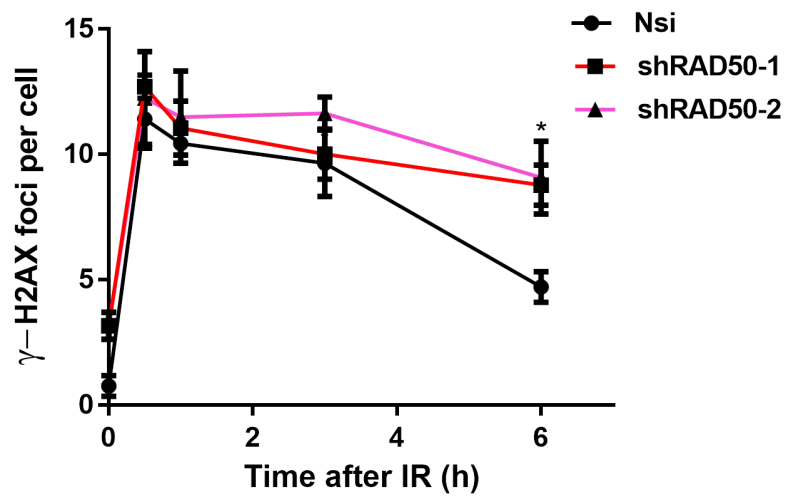

Figure 3. Effects of RAD50 knockdown on $\gamma$-H2AX foci formation after IR exposure. DNA damage was assessed by $\gamma$-H2AX foci staining. HCT116 (A) and HT29 (B) cells expressing non-silencing (Nsi) shRNA or RAD50 shRNA were irradiated with 2 Gy of $\gamma$-radiation, stained with $\gamma$-H2AX antibody at subsequent time intervals, and $\gamma-\mathrm{H} 2 \mathrm{AX}$ foci were counted. Data represent mean $\pm \mathrm{SD}$. Student's $\mathrm{t}-\mathrm{test},{ }^{*}, \mathrm{p}<0.05,{ }^{\star *}, \mathrm{p}<0.01$ compared to Nsi cells. To quantify the number of $\gamma-\mathrm{H} 2 \mathrm{AX}$ foci, at least 100 nuclei were analyzed from each sample.

A

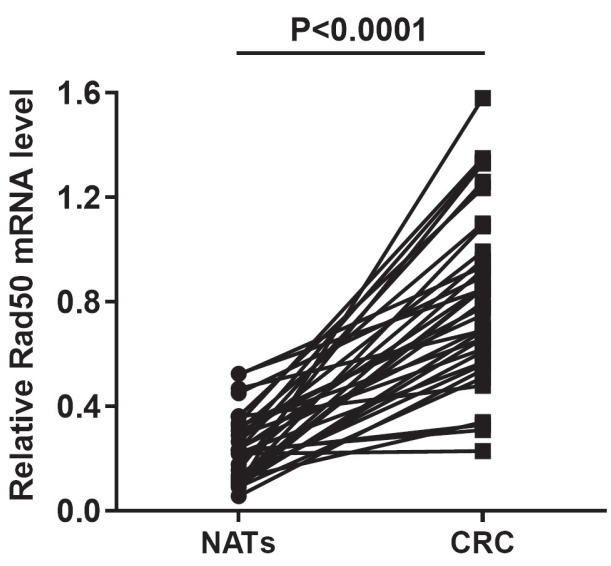

B

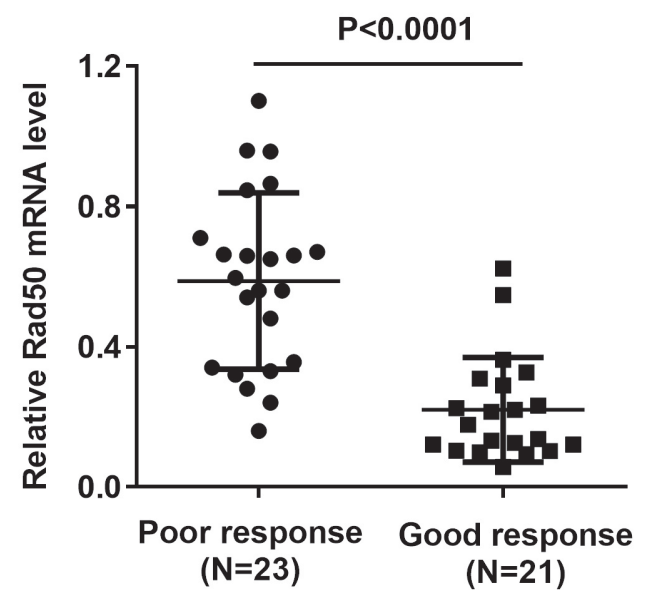

Figure 4. RAD50 expression is upregulated in CRC patients and positively associated with radioresistance in CRC patients. A. Quantitative PCR analysis of RAD50 mRNA expression in tissue samples from 36 pairs of CRC patients and matched NATs. B. Quantitative PCR analysis of RAD50 mRNA expression in tissue samples from CRC patients who showed good response $(n=21)$ or poor response $(n=23)$ to radiotherapy. 
An efficient DNA repair machinery is considered a hallmark of radioresistance. MRN complex is known to play a critical role in DNA repair and cell cycle regulation and components of this complex represent promising targets for radiosensitization [12]. Rhee et al. generated recombinant adenoviruses to interfere with Nbs1 function which enhances the radioresponse of head and neck tumors [13]. Transfection with Mre11 siRNA increases sensitivity of human tumor cells to heat radiation [14].

As a key member of this complex, malfunction of RAD50 reduces MRN complex-mediated DNA repair directly [15]. It has been reported that RAD50-deficient cells displayed defects in DNA damage-induced cell cycle regulation and DNA repair [16]. Disruption of RAD50 function by a recombinant adenovirus abrogates the DSB induced G2/M arrest and reduces the DNA repair capability in nasopharyngeal carcinoma cells [8]. In our present study, we introduced two independent shRNAs against RAD50 into two authentic CRC cell lines and monitored their response to irradiation. Consistent with previous reports in other cancer types, downregulation of RAD50 increased the sensitivity to irradiation in these two cell lines (Figure 1). DSBs repair failure initiates apoptosis by activating ATM (Ataxia telangiectasia mutated) kinase and the downstream pathway $[17,18]$. We observed that knockdown of RAD50 led to an increase in caspase activity, indicative of more apoptosis (Figure 2).

Phosphorylation of H2AX ( $\gamma-\mathrm{H} 2 \mathrm{AX})$ by ATM and ATR (ATM-Rad3-related) is the first step to recruit and localize repair proteins. $\gamma-\mathrm{H} 2 \mathrm{AX}$ can be used as a biomarker for DSBs, and its disappearance indicates the repair of the DNA damage $[19,20]$. We performed $\gamma-\mathrm{H} 2 \mathrm{AX}$ assay to evaluate the dynamics of DSBs repair after irradiation in our cells. Interestingly, RAD50 knockdown cells showed reduced DSB repair efficiency compared to control cells (Figure 3), thus may confer vulnerability to irradiation. This partially explains the increased sensitivity to irradiation in the RAD50 knockdown cellsMutations in the genes encoding MRN complex have been shown to associate with risk for breast cancer [21]. Expression levels of MRE11, RAD50 and NIBRIN protein are found to be significantly correlated with the postoperative survival in patients with pancreatic ductal adenocarcinoma [22]. Recently, RAD50 has been found to be dysregulated in premalignant immortalized human colonic epithelial cells and may be implicated in early stage of CRC development $[23,24]$. However, RAD50 expression and its association with patient survival have not been investigated in CRC. We observed that RAD50 was upregulated in CRC tumor tissues compared to NATs as well as in patients who did not respond to RT (Figure 4). RAD50 expression was significantly correlated with tumor response to RT and overall CRC patient survival (Figure 5 and Table 1). These results demonstrate that RAD50 may play a role in regulating radiosensitivity in CRC patients and serve as a predictive biomarker for RT.

In conclusion, our studies demonstrate that RAD50 is involved in radiosensitivity in CRC cells. RAD50 is upregu-

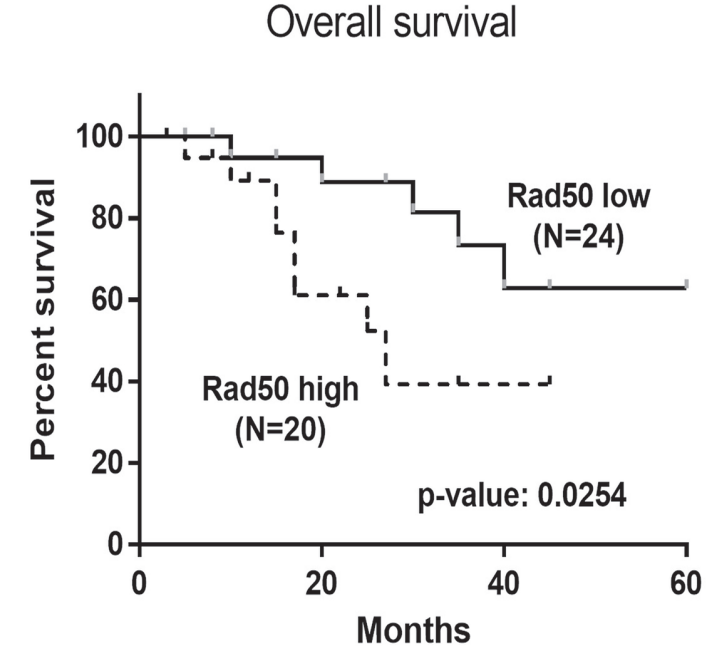

Figure 5. RAD50 expression is negatively associated with overall survival in CRC patients receiving radiotherapy. Survival curves showing the correlation of RAD50 with overall survival in CRC patients receiving radiotherapy $(\mathrm{p}=\mathbf{0 . 0 2 5 4})$.

Table 1. Correlation between RAD50 expression and tumor response to radiotherapy treatment according to TRG in CRC patients.

\begin{tabular}{cccc}
\hline RAD50 expression & Good response & Poor response & p-value \\
\hline Low & 17 & 7 & 0.001 \\
High & 4 & 16 & \\
\hline
\end{tabular}

Good response was defined as patients with TRG 4 whereas poor response was defined as patients with TRG $0,1,2$, and 3 .

lated in CRC cancerous tissues and especially in the patients who are resistant to RT. RAD50 expression is also associated with patient survival and therefore, it can serve as a biomarker for patient survival in CRC.

Acknowledgments: This study was supported by the Natural Science Foundation from Jiangsu Province (SBK2015020256).

\section{References}

[1] RASTOGI RP, RICHA, KUMAR A, TYAGI MB, SINHA RP. Molecular mechanisms of ultraviolet radiation-induced DNA damage and repair. J Nucleic Acids 2010; 2010: 592980. doi: $10.4061 / 2010 / 592980$

[2] GALANTY Y, BELOTSERKOVSKAYA R, COATES J, POLO S, MILLER KM et al. Mammalian SUMO E3-ligases PIAS1 and PIAS4 promote responses to DNA double-strand breaks. Nature 2009; 462: 935-939. doi: 10.1038/nature08657

[3] BEGG AC, STEWART FA, VENS C. Strategies to improve radiotherapy with targeted drugs. Nat Rev Cancer 2011; 11: 239-253. doi: 10.1038/nrc3007

[4] POWELL SN, BINDRA RS. Targeting the DNA damage response for cancer therapy. DNA Repair (Amst) 2009; 8: 1153-1165. doi: 10.1016/j.dnarep.2009.04.011 
[5] LAMARCHE BJ, ORAZIO NI, WEITZMAN MD. The MRN complex in double-strand break repair and telomere maintenance. FEBS Lett 2010; 584: 3682-3695. doi: 10.1016/j.febslet.2010.07.029

[6] WILLIAMS GJ, LEES-MILLER SP, TAINER JA. Mre11RAD50-Nbs1 conformations and the control of sensing, signaling, and effector responses at DNA double-strand breaks. DNA Repair (Amst) 2010; 9: 1299-1306. doi: 10.1016/j. dnarep.2010.10.001

[7] HOPFNER KP, CRAIG L, MONCALIAN G, ZINKEL RA, USUI T et al. The RAD50 zinc-hook is a structure joining Mre11 complexes in DNA recombination and repair. Nature 2002; 418: 562-566.

[8] CHANG L, HUANG J, WANG K, LI J, YAN R et al. Targeting RAD50 sensitizes human nasopharyngeal carcinoma cells to radiotherapy. BMC Cancer 2016; 16: 190. doi: 10.1186/ $\underline{\text { s12885-016-2190-8 }}$

[9] ABUZEID WM, JIANG X, SHI G, WANG H, PAULSON $D$ et al. Molecular disruption of RAD50 sensitizes human tumor cells to cisplatin-based chemotherapy. J Clin Invest 2009; 119: 1974-1985. doi: 10.1172/JCI33816

[10] THIES S, LANGER R. Tumor regression grading of gastrointestinal carcinomas after neoadjuvant treatment. Front Oncol 2013; 3: 262. doi: 10.3389/fonc. 2013.00262

[11] DENG R, TANG J, MA JG, CHEN SP, XIA LP et al. PKB/Akt promotes DSB repair in cancer cells through upregulating Mre11 expression following ionizing radiation. Oncogene 2011; 30: 944-955. doi: 10.1038/onc.2010.467

[12] CHAI W, SFEIR AJ, HOSHIYAMA H, SHAY JW, WRIGHT WE. The involvement of the Mre11/RAD50/Nbs1 complex in the generation of G-overhangs at human telomeres. EMBO Rep 2006; 7: 225-230.

[13] RHEE JG, LI D, SUNTHARALINGAM M, GUO C, O'MALLEY BW JR. et al. Radiosensitization of head/neck squamous cell carcinoma by adenovirus-mediated expression of the Nbs1 protein. Int J Radiat Oncol Biol Phys 2007; 67: 273-278.

[14] DYNLACHT JR, BATUELLO CN, LOPEZ JT, KIM KK, TURCHI JJ. Identification of Mre11 as a target for heat radiosensitization. Radiat Res 2011; 176: 323-332.
[15] WESTMORELAND J, MA W, YAN Y, VAN HULLE K, MALKOVA A et al. RAD50 is required for efficient initiation of resection and recombinational repair at random, gammainduced double-strand break ends. PLoS Genet 2009; 5: e1000656. doi: 10.1371/journal.pgen.1000656

[16] GATEI M, JAKOB B, CHEN P, KIJAS AW, BECHEREL OJ et al. ATM protein-dependent phosphorylation of RAD50 protein regulates DNA repair and cell cycle control. J Biol Chem 2011; 286: 31542-31556. doi: 10.1074/jbc.M111.258152

[17] DIFILIPPANTONIO S, CELESTE A, KRUHLAK MJ, LEE Y, DIFILIPPANTONIO MJ et al. Distinct domains in Nbs1 regulate irradiation-induced checkpoints and apoptosis. J Exp Med 2007; 204: 1003-1011.

[18] STRACKER TH, MORALES M, COUTO SS, HUSSEIN H, PETRINI JH. The carboxy terminus of NBS1 is required for induction of apoptosis by the MRE11 complex. Nature 2007; 447: 218-221.

[19] KUO LJ, YANG LX. Gamma-H2AX - a novel biomarker for DNA double-strand breaks. In Vivo 2008; 22: 305-309.

[20] LOBRICH M, SHIBATA A, BEUCHER A, FISHER A, ENSMINGER $M$ et al. gammaH2AX foci analysis for monitoring DNA double-strand break repair: strengths, limitations and optimization. Cell Cycle 2010; 9: 662-669.

[21] HSU HM, WANG HC, CHEN ST, HSU GC, SHEN CY et al. Breast cancer risk is associated with the genes encoding the DNA double-strand break repair Mre11/RAD50/ Nbs1 complex. Cancer Epidemiol Biomarkers Prev 2007; 16: 2024-2032.

[22] HORST K, GANZERA S, KAISERS W, MUNDING J, FLOTT-RAHMEL B et al. Influence of MRE11, RAD50 and NIBRIN protein expression on survival in pancreatic carcinoma after curative resection. Pathol Res Pract 2013; 209: 635-639. doi: 10.1016/j.prp.2013.07.003

[23] ZHANG L, KIM S, JIA G, BUHMEIDA A, DALLOL A et al. Exome Sequencing of Normal and Isogenic Transformed Human Colonic Epithelial Cells (HCECs) Reveals Novel Genes Potentially Involved in the Early Stages of Colorectal Tumorigenesis. BMC Genomics 2015; 16 Suppl 1: S8. doi: 10.1186/1471-2164-16-S1-S8

[24] ZHANG L, KOMUROV K, WRIGHT WE, SHAY JW. Identification of novel driver tumor suppressors through functional interrogation of putative passenger mutations in colorectal cancer. Int J Cancer 2013; 132: 732-737. doi: 10.1002/ijc.27705 